ESAIM: M2AN 47 (2013) 789-805

DOI: $10.1051 / \mathrm{m} 2 \mathrm{an} / 2012050$
ESAIM: Mathematical Modelling and Numerical Analysis

www.esaim-m2an.org

\title{
DUAL-MIXED FINITE ELEMENT METHODS FOR THE NAVIER-STOKES EQUATIONS *,**
}

\author{
Jason S. Howell ${ }^{1}$ AND Noel J. WALKington ${ }^{2}$
}

\begin{abstract}
A mixed finite element method for the Navier-Stokes equations is introduced in which the stress is a primary variable. The variational formulation retains the mathematical structure of the Navier-Stokes equations and the classical theory extends naturally to this setting. Finite element spaces satisfying the associated inf-sup conditions are developed.
\end{abstract}

Mathematics Subject Classification. 65N60, 65N12, 65M60, 65M12.

Received June 23, 2011. Revised July 11, 2012.

Published online March 29, 2013.

\section{INTRODUCTION}

The focus of this work is the development of mixed finite element schemes for the stationary Navier-Stokes equations where the fluid stress is a primary unknown of interest. The development of a corresponding scheme for the Stokes problem has been recently established [19]; however, this scheme only computes the symmetric part of the velocity gradient, so the extension to the Navier-Stokes equations is not direct since the convective term involves the full gradient. Below we propose a mixed method based upon the usual skew-symmetric formulation of the Navier-Stokes equations that allows for direct approximation of the stress and the velocity gradient. Specifically, we write the Navier-Stokes equations as

$$
\begin{gathered}
(1 / 2)(u . \nabla) u-\operatorname{div}(S)=f, \\
S=\mathcal{A}(\nabla u)-p I-(1 / 2) u \otimes u, \\
\operatorname{div}(u)=0,
\end{gathered}
$$

Here $\mathcal{A}(\nabla u)=\nu\left(\nabla u+(\nabla u)^{T}\right)$ is the "deviatoric" part of the stress, $p I$ is the hydrostatic stress, and the "Bernoulli" stress $(1 / 2) u \otimes u$ arises from the identity

$$
(u . \nabla) u=(1 / 2)(\nabla u) u+(1 / 2) \operatorname{div}(u \otimes u) \quad \text { when } \quad \operatorname{div}(u)=0 .
$$

Keywords and phrases. Navier-Stokes equations, mixed methods.

* Supported in part by National Science Foundation Grants DMS-1115228 This work was also supported by the NSF through the Center for Nonlinear Analysis.

** This material is partially based upon work supported by the Center for Nonlinear Analysis (CNA) under the National Science Foundation Grant No. DMS-0635983.

1 Department of Mathematics, College of Charleston, Charleston, 29424 SC. howelljs@cofc.edu

2 Department of Mathematics, Carnegie Mellon University, Pittsburgh, 15213 PA. noelw@andrew.cmu.edu 
The classical formulation $[8,17,27]$ is obtained by eliminating $S$ from equations (1). Existence of solutions to equations (1) will be established with the stress in $H\left(\Omega\right.$; div); in particular, $\operatorname{div}(S) \in L^{2}(\Omega)^{d}$ and this is only possible if $f \in L^{2}(\Omega)$. This additional regularity of the stress, and corresponding restriction on the data, is typical of mixed methods [8].

The central issue in any mixed formulation is the set of compatibility conditions between the spaces which are typically expressed as inf-sup conditions. In order to focus on these issues, and minimize peripheral technical detail, we will only consider the stationary problem with Dirichlet boundary data and the situation where $\mathcal{A}: \mathbb{R}^{d \times d} \rightarrow \mathbb{R}_{\mathrm{sym}}^{d \times d}$ is linear. However, the extension to include other boundary conditions, maximally monotone stress strain relations (which model viscoelastic fluids), and the evolution problem is direct.

The rest of this paper is organized as follows. The remainder of this section reviews related results, and the following section develops a variational formulation of equations (1) and establishes existence of solutions. In Section 3 finite element approximations are studied and standard error estimates are derived. Finite element spaces satisfying the crucial inf-sup properties are developed in Section 4 and a numerical example is presented in Section 5.

\subsection{Related results}

Traditional numerical methods for computing approximate solutions of fluid flows are based on the primitive velocity-pressure formulation, and (accurate) approximations of the stress must be computed via post-processing techniques such as $L^{2}$ projection or Superconvergent Patch Recovery [4,29-33]. In addition to the extra computational expense, these approximations of the fluid stress may suffer from instabilities and may not be appropriate for fluids with a complex microstructure, such as shear-thinning or viscoelastic fluids.

Mixed and dual-mixed formulations that include a stress-like quantity may be found in $[10,11,14-16]$. In this paper we address the following issues that have arisen in this context.

1. Often non-physical quantities such as the non-symmetric "pseudostress" $\sigma=\nu \nabla u-p I$ are introduced as primary variables $[10,11,14-16]$.

2. The constitutive relation $\mathcal{A}$ is often inverted $[10,11,14-16]$. Closed form expressions for the inverse may not be available for fluids exhibiting complex microstructure.

3. Often the mathematical structure of the Navier-Stokes equations is lost; for example, the skew symmetry of the nonlinear terms. This gives rise to a plethora of technical issues; examples include:

(a) Often the Hilbert space setting needs to be abandoned $[15,16]$.

(b) The classical energy estimate may not be available $[10,11,15,16]$.

(c) Elementary monotonicity arguments used for existence are not available and alternative arguments (e.g. BRR theory [9]) are required $[10,15,16]$.

For the evolutionary problem these issues can preclude long time existence of solutions.

The formulation presented below is unique in the sense that (a) the trace-free velocity gradient is a primary unknown, (b) the pressure is eliminated by proper definition of associated function spaces and can be recovered by a simple postprocessing calculation, (c) the underlying problem structure allows for nonlinear constitutive laws which will be of critical importance when approximating flows of non-Newtonian fluids, and (d) the skewsymmetrization of the nonlinear convective term gives straightforward proofs of existence and uniqueness results for the continuous and discrete variational problems. Additionally, the underlying structure of the scheme is related to many finite element methods for linear elasticity with weakly-imposed stress symmetry.

\section{VARIATIONAL FORMULATION}

Below $\Omega \subset \mathbb{R}^{d}, d=2,3$, is a bounded Lipschitz domain with boundary, and standard notation is used for the Lebesgue and Sobolev spaces. The pairing $(f, g)$ denotes the standard $L^{2}(\Omega)$ inner product for scalar, vector, and tensor functions $f$ and $g$. 
Assumption 2.1. There exist constants $C, \nu>0$ such that the constitutive relation $\mathcal{A}: \mathbb{R}^{d \times d} \rightarrow \mathbb{R}_{\mathrm{sym}}^{d \times d}$ satisfies

1. $\mathcal{A}(G)=\mathcal{A}\left(G^{\mathrm{sym}}\right)$, where $G^{\mathrm{sym}}=(1 / 2)\left(G+G^{\top}\right)$,

2. $(\mathcal{A}(G), G) \geq \nu\left\|G^{\mathrm{sym}}\right\|_{L^{2}(\Omega)}^{2}$, and

3. $(\mathcal{A}(G), H) \leq C\|G\|_{L^{2}(\Omega)}\|H\|_{L^{2}(\Omega)}$.

The dual-mixed formulation of the Navier-Stokes equations will be posed in the spaces:

$$
\begin{gathered}
\mathbb{G}=\left\{G \in L^{2}(\Omega)^{d \times d} \mid \operatorname{tr}(G)=0\right\} \\
\mathbb{U}=L^{2}(\Omega)^{d}, \\
\mathbb{S}=\left\{S \in L^{2}(\Omega)^{d \times d} \mid \operatorname{div}(S) \in L^{2}(\Omega)^{d} \text { and } \int_{\Omega} \operatorname{tr}(S)=0\right\} .
\end{gathered}
$$

Writing $G=\nabla u$, the incompressibility condition $\operatorname{div}(u)=0$ becomes $\operatorname{tr}(G)=0$; that is, $G \in \mathbb{G}$. The NavierStokes equations may then be posed as: $(G, u, S) \in \mathbb{G} \times \mathbb{U} \times \mathbb{S}$,

$$
\begin{aligned}
(\mathcal{A}(G), H)-(1 / 2)(u \otimes u, H)-(S, H) & =0, & H & \in \mathbb{G} \\
(1 / 2)(G u, v)-(\operatorname{div}(S), v) & =(f, v), & v & \in \mathbb{U} \\
(G, T)+(u, \operatorname{div}(T)) & =0, & T & \in \mathbb{S},
\end{aligned}
$$

To illustrate that this weak statement has the same structure as the usual formulation of the Navier-Stokes equations, we introduce the following bilinear and (skew-symmetric) trilinear forms.

Definition 2.2. With the spaces defined as in (2)

1. $a:(\mathbb{G} \times \mathbb{U})^{2} \rightarrow \mathbb{R}$,

2. $b: \mathbb{S} \times(\mathbb{G} \times \mathbb{U}) \rightarrow \mathbb{R}$,

$$
a((G, u),(H, v))=(\mathcal{A}(G), H) .
$$

$$
\begin{aligned}
& b(S,(H, v))=(S, H)+(\operatorname{div}(S), v) . \\
& \mathbb{Z}=\operatorname{Ker}\left(B^{T}\right)=\{(G, u) \in \mathbb{G} \times \mathbb{U} \mid b(T,(G, u))=0, T \in \mathbb{S}\} .
\end{aligned}
$$

3. $c:(\mathbb{G} \times \mathbb{U})^{3} \rightarrow \mathbb{R}$,

$$
c((F, w),(G, u),(H, v))=(1 / 2)[(G w, v)-((u \otimes w), H)]=(1 / 2)[(G w, v)-(H w, u)] .
$$

The dual-mixed formulation (3) then takes the classical form: $((G, u), S) \in(\mathbb{G} \times \mathbb{U}) \times \mathbb{S}$,

$$
\begin{aligned}
a((G, u),(H, v))+c((G, u),(G, u),(H, v))-b(S,(H, v)) & =F(H, v), & (H, v) & \in \mathbb{G} \times \mathbb{U} \\
b(T,(G, u)) & =0, & T & \in \mathbb{S} .
\end{aligned}
$$

\subsection{Well-posedness}

In this section it is shown that the classical analysis for the mixed formulation of the Navier-Stokes equations extends to the dual-mixed formulation (3). The following lemma originates from [2] and is useful when testing the stress with trace free functions $H \in \mathbb{G}$.

Lemma 2.3. Let $\Omega \subset \mathbb{R}^{d}$ be a bounded Lipschitz domain. If $S \in \mathbb{S}$, let $S_{0}=S-(1 / d) \operatorname{tr}(S) I$ denote the trace-free part of $S$. Then

$$
\|\operatorname{tr}(S)\|_{L^{2}(\Omega)} \leq C\left(\left\|S_{0}\right\|_{L^{2}(\Omega)}+\|\operatorname{div}(S)\|_{H^{-1}(\Omega)}\right) .
$$

In particular,

$$
\left\|S_{0}\right\|_{L^{2}(\Omega)}^{2}+\|\operatorname{div}(S)\|_{L^{2}(\Omega)}^{2} \leq\|S\|_{H(\Omega ; \operatorname{div})}^{2} \leq C\left(\left\|S_{0}\right\|_{L^{2}(\Omega)}^{2}+\|\operatorname{div}(S)\|_{L^{2}(\Omega)}^{2}\right),
$$

where $\|S\|_{H(\Omega ; \operatorname{div})}^{2} \equiv\|S\|_{L^{2}(\Omega)}^{2}+\|\operatorname{div}(S)\|_{L^{2}(\Omega)}^{2}$.

In the current context the Korn and Poincaré inequalities correspond to bounds upon $G^{\text {skw }}$ and $u$ by $G^{\text {sym }}$. 
Lemma 2.4. Let the spaces $\mathbb{G}, \mathbb{U}$ and $\mathbb{S}$ be the spaces characterized in equation (2), and let $\mathbb{Z} \subset \mathbb{G} \times \mathbb{U}$ be the Kernel introduced in Definition 2.2.

1. There exist constants $C, c>0$ such that

$$
\begin{array}{cr}
\sup _{(G, u) \in \mathbb{G} \times \mathbb{U}} \frac{(G, S)+(u, \operatorname{div}(S))}{\|(G, u)\|_{L^{2}(\Omega)}} \geq c\|S\|_{H(\Omega ; \operatorname{div})}, & S \in \mathbb{S} \\
\left\|\left(G^{\mathrm{skw}}, u\right)\right\|_{L^{2}(\Omega)} \leq C\left\|G^{\mathrm{sym}}\right\|_{L^{2}(\Omega)}, & (G, u) \in \mathbb{Z} .
\end{array}
$$

2. If $(G, u) \in \mathbb{Z}$ then $\|u\|_{L^{6}(\Omega)} \leq C\left\|G^{\mathrm{sym}}\right\|_{L^{2}(\Omega)} ;$ moreover, if $\left\{\left(G_{n}, u_{n}\right)\right\}_{n=0}^{\infty} \subset \mathbb{Z}$ and $G_{n} \rightarrow G$ in $L^{2}(\Omega){ }^{d \times d}$ then $u_{n} \rightarrow u$ in $L^{p}(\Omega)^{d}$ for $1 \leq p<6$.

The inf-sup condition follows directly upon selecting $(G, u)=\left(S_{0}, \operatorname{div}(S)\right)$ and appealing to the previous lemma. If $(G, u) \in \mathbb{Z}$, then $u \in H_{0}^{1}(\Omega)$ with $G=\nabla u$ and $\operatorname{div}(u)=0$ so the second assertion follows from the Korn and Poincaré inequalities and the Sobolev embedding theorem.

Corollary 2.5. Let $a(.,),. b(.,$.$) and c(., .,$.$) be the functions and \mathbb{Z}$ be the Kernel introduced in Definition 2.2.

1. $a(.,$.$) and b(.,$.$) are continuous and a(.,$.$) is coercive on \mathbb{Z}$;

$$
a((G, u),(G, u)) \geq \nu\left\|G^{\mathrm{sym}}\right\|_{L^{2}(\Omega)}^{2} \geq c_{a}\|(G, u)\|^{2} \quad(G, u) \in \mathbb{Z},
$$

where $c_{a}=\nu /\left(1+C^{2}\right)$.

2. $c: \mathbb{Z} \times \mathbb{Z} \rightarrow(\mathbb{G} \times \mathbb{U})^{\prime}$ is weakly continuous.

The following theorem establishes existence for the continuous problem (3), and will also provide existence of solutions for the numerical scheme.

Theorem 2.6. Let $\mathbb{G}, \mathbb{U}$ and $\mathbb{S}$ be separable reflexive Banach spaces, $a:(\mathbb{G} \times \mathbb{U})^{2} \rightarrow \mathbb{R}$ and $b: \mathbb{S} \times(\mathbb{G} \times \mathbb{U}) \rightarrow \mathbb{R}$ be bilinear and continuous. Let

$$
\mathbb{Z}=\{(G, u) \in \mathbb{G} \times \mathbb{U} \mid b(T,(G, u))=0, T \in \mathbb{S}\},
$$

and $c: \mathbb{Z}^{2} \times(\mathbb{G} \times \mathbb{U}) \rightarrow \mathbb{R}$ be trilinear and continuous. Assume

1. $a(.,$.$) is coercive on \mathbb{Z}: a((G, u),(G, u)) \geq c_{a}\|(G, u)\|^{2}$ for all $(G, u) \in \mathbb{Z}$.

2. $b(.,$.$) satisfies the inf-sup condition$

$$
\sup _{(G, u) \in \mathbb{G} \times \mathbb{U}} \frac{b(S,(G, u))}{\|(G, u)\|} \geq c_{b}\|S\|_{\mathbb{S}}, \quad S \in \mathbb{S} .
$$

3. $c((G, u),(G, u),(G, u))=0$ for all $(G, u) \in \mathbb{Z}$, and the map $c: \mathbb{Z} \times \mathbb{Z} \rightarrow(\mathbb{G} \times \mathbb{U})^{\prime}$ is weakly continuous.

Then for each $F \in(\mathbb{G} \times \mathbb{U})^{\prime}$ there exists $(G, u, S) \in \mathbb{G} \times \mathbb{U} \times \mathbb{S}$ such that

$$
\begin{array}{rlrl}
a((G, u),(H, v))+c((G, u),(G, u),(H, v))-b(S,(H, v)) & =F(H, v), & (H, v) & \in \mathbb{G} \times \mathbb{U}, \\
b(T,(G, u)) & =0 & T & \in \mathbb{S} .
\end{array}
$$

Moreover, $\|(G, u)\| \leq\left(1 / c_{a}\right)\|F\|$ and $\|S\|_{\mathbb{S}} \leq\left(1 / c_{b}\right)\left(1+C_{a} / c_{a}+\left(C_{c} / c_{a}^{2}\right)\|F\|\right)\|F\|$ where $C_{a}$ and $C_{c}$ denote continuity constants of $a(.,$.$) and c(., .,$.$) respectively.$ 
Proof. Let $\mathcal{F}: \mathbb{Z} \rightarrow \mathbb{Z}$ be characterized by

$$
(\mathcal{F}(G, u),(H, v))=a((G, u),(H, v))+c((G, u),(G, u),(H, v))-F(H, v), \quad(H, v) \in \mathbb{Z},
$$

where the pairing on the left is the inner product in $\mathbb{G} \times \mathbb{U}$. Setting $(H, v)=(G, u) \in \mathbb{Z}$ shows

$$
(\mathcal{F}(G, u),(G, u))=a((G, u),(G, u))-F(G, u) \geq c_{a}\|(G, u)\|^{2}-F(G, u),
$$

It follows [24], Corollary II.2.2, that $\mathcal{F}(G, u)=0$ for some $(G, u) \in \mathbb{Z}$ with norm $\|(G, u)\| \leq\left(1 / c_{a}\right)\|F\|$.

Existence of a stress follows from the continuity and coercivity of $b(.,$.$) on \mathbb{S} \times(\mathbb{G} \times \mathbb{U}) / \mathbb{Z}$. Specifically, if $(G, u) \in \mathbb{Z}$ satisfies $\mathcal{F}(G, u)=0$, then the mapping

$$
(H, v) \mapsto a((G, u),(H, v))+c((G, u),(G, u),(H, v))-F(H, v)
$$

vanishes on $\mathbb{Z}$, so is in the dual of $(\mathbb{G} \times \mathbb{U}) / \mathbb{Z}$. It follows that the problem; $S \in \mathbb{S}$,

$$
b(S,(H, v))=a((G, u),(H, v))+c((G, u),(G, u),(H, v))-F(H, v), \quad(H, v) \in \mathbb{G} \times \mathbb{U},
$$

has a unique solution; moreover

$$
c_{b}\|S\|_{\mathbb{S}} \leq C_{a}\|(G, u)\|+C_{c}\|(G, u)\|^{2}+\|F\| \leq\left(1+C_{a} / c_{a}+C_{c} / c_{a}^{2}\|F\|\right)\|F\| .
$$

\section{Finite Element approximation}

Let $\mathbb{G} \times \mathbb{U} \times \mathbb{S}$ be the spaces defined in (2) and $\mathbb{G}_{h} \times \mathbb{U}_{h} \times \mathbb{S}_{h}$ be (finite element) subspaces. The discrete problem corresponding to the variational form (3) is: $\left(G_{h}, u_{h}, S_{h}\right) \in \mathbb{G}_{h} \times \mathbb{U}_{h} \times \mathbb{S}_{h}$,

$$
\begin{aligned}
& \left(A\left(G_{h}\right), H_{h}\right)-(1 / 2)\left(u_{h} \otimes u_{h}, H_{h}\right)-\left(S_{h}, H_{h}\right)=0, \quad H_{h} \in \mathbb{G}_{h} \\
& (1 / 2)\left(G_{h} u_{h}, v_{h}\right)-\left(\operatorname{div}\left(S_{h}\right), v_{h}\right)=\left(f, v_{h}\right), \quad v_{h} \in \mathbb{U}_{h} \\
& \left(G_{h}, T_{h}\right)+\left(u_{h}, \operatorname{div}\left(T_{h}\right)\right)=0, \quad T_{h} \in \mathbb{S}_{h} .
\end{aligned}
$$

Using the functions $a(.,),. b(.,$.$) and c(., . .$.$) in Definition 2.2$ the discrete weak problem can be written as: $\left(G_{h}, u_{h}, S_{h}\right) \in \mathbb{G}_{h} \times \mathbb{U}_{h} \times \mathbb{S}_{h}$,

$$
\begin{aligned}
a\left(\left(G_{h}, u_{h}\right),\left(H_{h}, v_{h}\right)\right)+c\left(\left(G_{h}, u_{h}\right),\left(G_{h}, u_{h}\right),\left(H_{h}, v_{h}\right)\right)+b\left(S_{h},\left(H_{h}, v_{h}\right)\right) & =F\left(H_{h}, v_{h}\right), \\
b\left(T_{h},\left(G_{h}, u_{h}\right)\right) & =0,
\end{aligned}
$$

for $\left(H_{h}, v_{h}\right) \in \mathbb{G}_{h} \times \mathbb{U}_{h}$ and $T_{h} \in \mathbb{S}_{h}$.

In order for the discrete problem to be well-posed the discrete spaces need to inherit the inf-sup and Korn/Poincaré estimates stated in Lemma 2.4.

Assumption 3.1. There exist constants $c_{b}$ and $C>0$ independent of $h$ such that

$$
\begin{aligned}
& \sup _{\left(G_{h}, u_{h}\right) \in \mathbb{G}_{h} \times \mathbb{U}_{h}} \frac{\left(G_{h}, S_{h}\right)+\left(u_{h}, \operatorname{div}\left(S_{h}\right)\right)}{\left\|\left(G_{h}, u_{h}\right)\right\|_{L^{2}(\Omega)}} \geq c_{b}\left\|S_{h}\right\|_{H(\Omega ; \operatorname{div})}, \quad T \in \mathbb{S}_{h}, \\
& \left\|\left(G_{h}^{\mathrm{skw}}, u_{h}\right)\right\|_{L^{2}(\Omega)} \leq C\left\|G^{\mathrm{sym}}\right\|_{L^{2}(\Omega)} \quad\left(G_{h}, u_{h}\right) \in \mathbb{Z}_{h} .
\end{aligned}
$$

where $\mathbb{Z}_{h}=\left\{\left(G_{h}, u_{h}\right) \in \mathbb{G}_{h} \times \mathbb{U}_{h} \mid\left(G_{h}, S_{h}\right)+\left(u_{h}, \operatorname{div}\left(S_{h}\right)\right)=0, \quad S_{h} \in \mathbb{S}_{h}\right\}$. 


\subsection{Discrete weak problem}

Existence of a solution to the discrete problem will follow from Theorem 2.6 whenever the discrete spaces inherit the inf-sup condition (5) and discrete Korn inequality (6), since continuity of the trilinear form $c(., .,$. is immediate on finite dimensional spaces.

Lemma 3.2. Let $\left(\mathbb{G}_{h}, \mathbb{U}_{h}, \mathbb{S}_{h}\right) \subset(\mathbb{G}, \mathbb{U}, \mathbb{S})$ be a finite dimensional subspace satisfying Assumption 3.1. Then there exists $\left(G_{h}, u_{h}, S_{h}\right) \in\left(\mathbb{G}_{h}, \mathbb{U}_{h}, \mathbb{S}_{h}\right)$ satisfying equations (4) such that

$$
\left\|G_{h}\right\|_{L^{2}(\Omega)}+\left\|u_{h}\right\|_{L^{2}(\Omega)} \leq\left(1 / c_{a}\right)\|f\|_{L^{2}(\Omega)} .
$$

and

$$
\left\|S_{h}\right\|_{H(\Omega ; \operatorname{div})} \leq\left(1 / c_{b}\right)\left(1+C_{a} / c_{a}+\left(\left\|c_{h}\right\| / c_{a}^{2}\right)\|f\|_{L^{2}(\Omega)}\right)\|f\|_{L^{2}(\Omega)},
$$

where $\left\|c_{h}\right\|$ is the norm of $c(., .,$.$) on \mathbb{Z}_{h} \times \mathbb{Z}_{h} \times\left(\mathbb{G}_{h} \times \mathbb{U}_{h}\right)$.

\subsection{Error estimates}

The following analogue of Lemma 2.4 establishes the compactness and embedding properties of the discrete spaces necessary to control the trilinear form $c(., .,$.$) .$

Lemma 3.3. Let $\left\{\left(\mathbb{G}_{h}, \mathbb{U}_{h}, \mathbb{S}_{h}\right)\right\}_{h>0}$ be a family of finite element subspaces of $(\mathbb{G}, \mathbb{U}, \mathbb{S})$ constructed over a regular family of triangulations of $\Omega$ satisfying the hypotheses of Assumption 3.1.

1. If $\left(G_{h}, u_{h}\right) \in \mathbb{Z}_{h}$ and $G_{h} \rightarrow G$ in $L^{2}(\Omega)$, then $u_{h} \rightarrow u$ in $L^{2}(\Omega)$.

2. If the triangulations are quasi-uniform there exists $C$ independent of $h$ such that $\left\|u_{h}\right\|_{L^{6}(\Omega)} \leq C\left\|G_{h}^{\mathrm{sym}}\right\|_{L^{2}(\Omega)}$ for $\left(G_{h}, u_{h}\right) \in \mathbb{Z}_{h}$.

Proof. Fix $\left(G_{h}, u_{h}\right) \in \mathbb{Z}_{h}$ and let $(\tilde{G}, \tilde{u}, \tilde{S}) \in \mathbb{G} \times \mathbb{U} \times \mathbb{S}$ satisfy

$$
\begin{array}{rlrl}
(\tilde{G}, H)-(v, \operatorname{div}(\tilde{S}))-(\tilde{S}, H) & =\left(G_{h}, H\right) & (H, v) & \in \mathbb{G} \times \mathbb{U} \\
(\tilde{u}, \operatorname{div}(T))+(\tilde{G}, T) & =0 & T & \in \mathbb{S} .
\end{array}
$$

Then $\tilde{u} \in H_{0}^{1}(\Omega), \nabla \tilde{u}=\tilde{G}$, and $\|\tilde{G}\|_{L^{2}(\Omega)} \leq\left\|G_{h}\right\|_{L^{2}(\Omega)}$. The Poincaré inequality and the Sobolev embedding theorem (in three dimensions) then show $\|\tilde{u}\|_{L^{6}(\Omega)} \leq C\left\|G_{h}\right\|_{L^{2}(\Omega)}$. Notice that $\left(G_{h}, u_{h}, 0\right)$ satisfies the discrete version of this equation, so classical finite element theory shows

$$
\left\|\tilde{u}-u_{h}\right\|_{L^{2}(\Omega)} \leq C\|\nabla \tilde{u}\|_{L^{2}(\Omega)} h \leq C\left\|G_{h}\right\|_{L^{2}(\Omega)} h .
$$

If $I_{h}: H^{1}(\Omega) \rightarrow \mathbb{U}_{h}$ denotes the Clément interpolant [12], the bound on $\left\|u_{h}\right\|_{L^{6}(\Omega)}$ follows from classical inverse estimates and approximation properties of $I_{h}$

$$
\left\|u_{h}\right\|_{L^{6}(\Omega)} \leq\left\|I_{h} \tilde{u}\right\|_{L^{6}(\Omega)}+\left\|I_{h} \tilde{u}-u_{h}\right\|_{L^{6}(\Omega)} \leq C\left\|I_{h} \tilde{u}\right\|_{H^{1}(\Omega)}+(1 / h)\left\|I_{h} \tilde{u}-u_{h}\right\|_{L^{2}(\Omega)} \leq C\|\tilde{u}\|_{H^{1}(\Omega)} .
$$

As with the Navier-Stokes equations [20], solutions are unique when $f$ is sufficiently small, and the discrete problem exhibits optimal rates of convergence.

Theorem 3.4. Let $\Omega \subset \mathbb{R}^{d}$ have Lipschitz continuous boundary and let $\left\{\left(\mathbb{G}_{h}, \mathbb{U}_{h}, \mathbb{S}_{h}\right)\right\}_{h>0}$ be a family of finite element subspaces of $(\mathbb{G}, \mathbb{U}, \mathbb{S})$ constructed over a regular family of quasi-uniform triangulations of $\Omega$ satisfying the hypotheses of Assumption 3.1. Assume $(G, u, S) \in \mathbb{G} \times \mathbb{U} \times \mathbb{S}$ satisfies equations (3) and $\left(G_{h}, u_{h}, S_{h}\right) \in$ $\mathbb{G}_{h} \times \mathbb{U}_{h} \times \mathbb{S}_{h}$ satisfies equations (4). If $\|f\|_{L^{2}(\Omega)}$ is sufficiently small there is a constant $C$, independent of $h$, such that

$$
\begin{aligned}
\left\|G-G_{h}\right\|_{L^{2}(\Omega)}+\left\|u-u_{h}\right\|_{L^{2}(\Omega)} & +\left\|S-S_{h}\right\|_{H(\Omega ; \operatorname{div})} \\
& \leq C\left\{\inf _{H_{h} \in \mathbb{G}_{h}}\left\|G-H_{h}\right\|_{L^{2}(\Omega)}+\inf _{v_{h} \in U_{h}}\left\|u-v_{h}\right\|_{L^{2}(\Omega)}+\inf _{T_{h} \in \mathbb{S}_{h}}\left\|S-T_{h}\right\|_{H(\Omega ; \operatorname{div})}\right\} .
\end{aligned}
$$


Proof. The proof is similar to the standard approach used for the classical mixed formulation of the NavierStokes equations [20], and will only be sketched here. The Galerkin orthogonality condition becomes

$$
\begin{array}{r}
a\left(\left(G-G_{h}, u-u_{h}\right),\left(H_{h}, v_{h}\right)\right)+b\left(S-S_{h},\left(H_{h}, v_{h}\right)\right) \\
=c\left(\left(G_{h}, u_{h}\right),\left(G_{h}, u_{h}\right),\left(H_{h}, v_{h}\right)\right)-c\left((G, u),(G, u),\left(H_{h}, v_{h}\right)\right),
\end{array}
$$

for all $\left(H_{h}, v_{h}\right) \in \mathbb{G}_{h} \times \mathbb{U}_{h}$. Fix $\left(G_{p}, u_{p}\right) \in \mathbb{Z}_{h}$ and write

$$
(E, e) \equiv\left(G-G_{h}, u-u_{h}\right)=\left(G-G_{p}, u-u_{p}\right)+\left(G_{p}-G_{h}, u_{p}-u_{h}\right) \equiv\left(E_{p}, e_{p}\right)+\left(E_{h}, e_{h}\right) .
$$

Setting $\left(H_{h}, v_{h}\right)=\left(E_{h}, e_{h}\right) \in \mathbb{Z}_{h}$ and using the coercivity of $a(.,$.$) on \mathbb{Z}_{h}$ and skew-symmetry of $c(., .,$.$) it$ follows that

$$
c\left\|\left(E_{h}, e_{h}\right)\right\|_{L^{2}(\Omega)} \leq C\left(\left\|\left(E_{p}, e_{p}\right)\right\|_{L^{2}(\Omega)}+\left\|S-T_{h}\right\|_{H(\Omega ; \operatorname{div})}+\|(G, u)\|_{L^{2}(\Omega)}\left\|\left(E_{h}, e_{h}\right)\right\|_{L^{2}(\Omega)}\right),
$$

where $T_{h} \in \mathbb{S}_{h}$ is arbitrary. When $\|(G, u)\|_{L^{2}(\Omega)} \leq C\|f\|_{L^{2}(\Omega)}$ is sufficiently small, the last term on the right can be absorbed into the left to show

$$
\begin{aligned}
\|(E, e)\|_{L^{2}(\Omega)} & \leq C\left(\inf _{\left(G_{p}, u_{p}\right) \in \mathbb{Z}_{h}}\left\|\left(G-G_{p}, u-u_{p}\right)\right\|_{L^{2}(\Omega)}+\inf _{T_{h} \in \mathbb{S}_{h}}\left\|S-T_{h}\right\|_{H(\Omega ; \text { div })}\right) \\
& \leq C\left(\inf _{\left(H_{h}, v_{h}\right) \in \mathbb{G}_{h} \times \mathbb{U}_{h}}\left\|\left(G-H_{h}, u-v_{h}\right)\right\|_{L^{2}(\Omega)}+\inf _{T_{h} \in \mathbb{S}_{h}}\left\|S-T_{h}\right\|_{H(\Omega ; \text { div })}\right),
\end{aligned}
$$

where the last line follows from the property that the discrete kernels $\mathbb{Z}_{h}$ optimally approximate $\mathbb{Z}$ when $b(.,$. satisfies the inf-sup condition. The error estimate for the stress now follows from the orthogonality relation and the inf-sup property of $b(.,$.$) .$

\section{Finite element spaces}

This section considers the development of finite element subspaces satisfying the crucial inf-sup condition in Assumption 3.1. Lemma 4.1 below provides several equivalent formulations of the inf-sup condition useful for this task. This lemma shows that if $\mathbb{G}_{h} \times \mathbb{U}_{h} \times \mathbb{S}_{h}$ satisfies Assumption 3.1, then the space $\mathbb{G}_{h}^{\text {skw }} \times \mathbb{U}_{h} \times \mathbb{S}_{h}$ is a stable space for the elasticity problem with weak symmetry $[3,5,8]$; here $\mathbb{G}_{h}^{\text {skw }}$ denotes the subspace of skew matrices in $\mathbb{G}_{h}$. However, this is not sufficient; an additional property is required if Assumption 3.1 is to hold. In Section 4.2 it is shown that in two dimensions the finite element spaces developed for the elasticity problem will typically inherit the additional requirement; however, this is not so in three dimensions. This issue is circumvented in Section 4.3 which develops a new family of elements satisfying Assumption 3.1 in two and three dimensions.

The following lemma reformulates the inf-sup condition of Assumption 3.1 into a form more amenable to analysis using macroelement techniques.

Lemma 4.1. Let $\mathbb{G} \subset L^{2}(\Omega)^{d \times d}$ be closed under transpose, $\mathbb{U} \subset L^{2}(\Omega)^{d}$, and $\mathbb{S} \subset H(\operatorname{div}, \Omega)^{d \times d}$ be closed subspaces. Let

$$
\begin{aligned}
\mathbb{Z} & =\{(G, u) \in \mathbb{G} \times \mathbb{U} \mid(G, T)+(u, \operatorname{div}(T))=0, T \in \mathbb{S}\}, \\
Z & =\{T \in \mathbb{S} \mid(u, \operatorname{div}(T))=0, u \in \mathbb{U}\}, \\
Z^{\mathrm{sym}} & =\left\{T \in \mathbb{S} \mid\left(G^{\mathrm{skw}}, T\right)+(u, \operatorname{div}(T))=0,\left(G^{\mathrm{skw}}, u\right) \in \mathbb{G}^{\mathrm{skw}} \times \mathbb{U}\right\},
\end{aligned}
$$


where $\mathbb{G}=\mathbb{G}^{\mathrm{skw}} \oplus \mathbb{G}^{\mathrm{sym}}$ is the decomposition of $\mathbb{G}$ into skew-symmetric and symmetric matrices. Then the following are equivalent:

1. There exist constants $c$ and $C>0$ such that

$$
\begin{array}{crl}
\sup _{(G, u) \in \mathbb{G} \times \mathbb{U}} \frac{(G, T)+(u, \operatorname{div}(T))}{\|(G, u)\|_{L^{2}(\Omega)}} \geq c\|T\|_{H(\Omega ; \operatorname{div})}, & T \in \mathbb{S}, \\
\left\|\left(G^{\mathrm{skw}}, u\right)\right\|_{L^{2}(\Omega)} \leq C\left\|G^{\mathrm{sym}}\right\|_{L^{2}(\Omega)}, & (G, u) \in \mathbb{Z} .
\end{array}
$$

2. There exists a constant $c>0$ such that

$$
\begin{array}{cc}
\sup _{T \in \mathbb{S}} \frac{\left(G^{\mathrm{skw}}, T\right)+(u, \operatorname{div}(T))}{\|T\|_{H(\Omega ; \operatorname{div})}} \geq c\left\|\left(G^{\mathrm{skw}}, u\right)\right\|_{L^{2}(\Omega)}, & \left(G^{\mathrm{skw}}, u\right) \in \mathbb{G}^{\mathrm{skw}} \times \mathbb{U}, \\
\sup _{G^{\mathrm{sym}} \in \mathbb{G}^{\text {sym }}} \frac{\left(G^{\mathrm{sym}}, T\right)}{\left\|G^{\mathrm{sym}}\right\|_{L^{2}(\Omega)}} \geq c\|T\|_{H(\Omega ; \operatorname{div})}, & T \in Z^{\mathrm{sym}} .
\end{array}
$$

3. There exists a constant $c>0$ such that

$$
\begin{array}{cr}
\sup _{T \in \mathbb{S}} \frac{(u, \operatorname{div}(T))}{\|T\|_{H(\Omega ; \operatorname{div})}} \geq c\|u\|_{L^{2}(\Omega)}, & u \in \mathbb{U}, \\
\sup _{T \in Z} \frac{\left(G^{\mathrm{skw}}, T\right)}{\|T\|_{H(\Omega ; \operatorname{div})}} \geq c\left\|G^{\mathrm{skw}}\right\|_{L^{2}(\Omega)}, & G^{\mathrm{skw}} \in \mathbb{G}^{\mathrm{skw}}, \\
\sup _{G^{\mathrm{sym}} \in \mathbb{G}^{\text {sym }}} \frac{\left(G^{\mathrm{sym}}, T\right)}{\left\|G^{\mathrm{sym}}\right\|_{L^{2}(\Omega)}} \geq c\|T\|_{H(\Omega ; \operatorname{div})}, & T \in Z^{\mathrm{sym}} .
\end{array}
$$

\section{Remark 4.2.}

1. Note that tensors in $Z^{\text {sym }}$ are only "weakly symmetric", i.e., they need not be symmetric pointwise.

2. The first condition of (2), or equivalently the first two conditions of (3), are the stability conditions for the elasticity problem with weak symmetry. The development of stable spaces for this problem can be found in $[1,3,6,13,18,26]$.

3. The last condition of (2) and (3) is necessary to compute the full gradient, G. Spaces developed for the elasticity problem will only compute the symmetric part of the gradient if this condition fails.

4. $\|T\|_{H(\Omega ; \operatorname{div})}=\|T\|_{L^{2}(\Omega)}$ for tensors $T \in Z^{\mathrm{sym}}$.

Proof. The hypothesis that $\mathbb{G}$ is closed under transpose allows $\mathbb{G}$ to be decomposed into a direct sum of skew and symmetric matrices, $\mathbb{G}=\mathbb{G}^{\mathrm{skw}} \oplus \mathbb{G}^{\mathrm{sym}}$. Then writing

$$
b_{1}\left(T, G^{\mathrm{sym}}\right)+b_{2}\left(T,\left(G^{\mathrm{skw}}, u\right)\right)=\left(G^{\mathrm{sym}}, T\right)+\left(G^{\mathrm{skw}}, T\right)+(u, \operatorname{div}(T)),
$$

the equivalence of conditions (1) and (2) follows from the equivalence of conditions (1) and (2) in [19], Theorem 3.2.

The equivalence of conditions (1) and (3) in [19], Theorem 3.1 shows that the inf-sup condition in equation (8) is equivalent to

$$
\begin{array}{lrl}
\sup _{T \in \mathbb{S}} \frac{(u, \operatorname{div}(T))}{\|T\|_{H(\Omega ; \operatorname{div})}} \geq c\|u\|_{L^{2}(\Omega)}, & u \in \mathbb{U}, \\
\sup _{T \in Z} \frac{\left(G^{\text {skw }}, T\right)}{\|T\|_{H(\Omega ; \operatorname{div})}} \geq c\left\|G^{\text {skw }}\right\|_{L^{2}(\Omega)}, & G^{\text {skw }} \in \mathbb{G}^{\text {skw }} .
\end{array}
$$




\subsection{Macroelement construction}

The following notation facilitates a unified discussion of the two and three dimensional situation.

\section{Notation 4.3.}

1. If $\mathbb{G}_{h}^{\text {skw }} \times \mathbb{U}_{h} \times \mathbb{S}_{h}$ is a subspace of $\mathbb{G}^{\mathrm{skw}} \times \mathbb{U} \times \mathbb{S}$, then $\mathbb{Z}_{h}, Z_{h}$, and $Z_{h}^{\mathrm{sym}}$ denote the analogues of the spaces $\mathbb{Z}, Z$, and $Z^{\mathrm{sym}}$ defined in (7), respectively.

2. When $d=2$, if $a: \Omega \rightarrow \mathbb{R}$ and $\psi: \Omega \rightarrow \mathbb{R}^{2}$ then

$$
W(a)=\left[\begin{array}{cc}
0 & a \\
-a & 0
\end{array}\right], \quad \operatorname{Curl}(\psi)=\left[\begin{array}{ll}
-\psi_{1, y} & \psi_{1, x} \\
-\psi_{2, y} & \psi_{2, x}
\end{array}\right] .
$$

If $V_{h} \subset H^{1}(\Omega)$, then $\operatorname{Curl}\left(V_{h}\right)=\left\{\operatorname{Curl}(\psi) \mid \psi \in V_{h}^{2}\right\}$.

3. When $d=3$, if $a: \Omega \rightarrow \mathbb{R}^{3}$ and $\psi: \Omega \rightarrow \mathbb{R}^{3 \times 3}$ then

$$
W(a)=\left[\begin{array}{ccc}
0 & -a_{3} & a_{2} \\
a_{3} & 0 & -a_{1} \\
-a_{2} & a_{1} & 0
\end{array}\right], \quad \operatorname{Curl}(\psi)=\left[\begin{array}{ccc}
\psi_{13, y}-\psi_{12, z} & \psi_{11, z}-\psi_{13, x} & \psi_{12, x}-\psi_{11, y} \\
\psi_{23, y}-\psi_{22, z} & \psi_{21, z}-\psi_{23, x} & \psi_{22, x}-\psi_{21, y} \\
\psi_{33, y}-\psi_{32, z} & \psi_{31, z}-\psi_{33, x} & \psi_{32, x}-\psi_{31, y}
\end{array}\right] .
$$

If $V_{h} \subset H^{1}(\Omega)$, then $\operatorname{Curl}\left(V_{h}\right)=\left\{\operatorname{Curl}(\psi) \mid \psi \in V_{h}^{3 \times 3}\right\}$.

If $\mathbb{G}_{h}^{\mathrm{skw}} \times \mathbb{U}_{h} \times \mathbb{S}_{h}$ is a stable triple of finite element spaces for the elasticity problem with weak symmetry, the macroelement technique $[8,25]$ can be used to establish the last condition in item 3 of Lemma 4.1 by showing that the only tensors $S_{h} \in Z^{\text {sym }}$ orthogonal to $\mathbb{G}_{h}^{\text {sym }}$ on a macroelement take the form $S_{h}=\delta I$ for $\delta \in \mathbb{R}$.

If $S_{h} \in Z_{h}^{\text {sym }}$ we suppose the subspace $\mathbb{G}_{h}^{\text {sym }}$ of symmetric trace-free matrix valued functions is sufficiently large to guarantee

$$
\int_{M} S_{h}: G_{h}^{\mathrm{sym}}=0, \quad G_{h}^{\mathrm{sym}} \in \mathbb{G}_{h}^{\mathrm{sym}} \quad \Rightarrow \quad S_{h}=\delta I+W(a) \text { on } M,
$$

for each macroelement. If $a \equiv 0$ and $\delta \in \mathbb{R}$ for tensors in $Z_{h}^{\text {sym }}$ with this structure, the macroelement methodology then shows

$$
\sup _{G_{h}^{\text {sym }} \in \mathbb{G}_{h}^{\text {sym }}} \frac{\left(G_{h}^{\mathrm{sym}}, S_{h}\right)}{\left\|G_{h}^{\mathrm{sym}}\right\|_{L^{2}(\Omega)}} \geq c\left\|S_{h}\right\|_{L^{2}(\Omega) / \mathbb{R}}, \quad S_{h} \in Z_{h}^{\mathrm{sym}} .
$$

The following line of argument will be used for this last step.

1. If $n$ is the normal to a common $(d-1)$ face of two finite elements of $M$, the jump, $\left[S_{h}\right] n$, of the normal component of $S_{h}$ vanishes.

(a) In two dimensions

$$
\left[S_{h}\right] n=[\delta] n-[a] n^{\perp} \quad \text { where } \quad\left(n_{1}, n_{2}\right)^{\perp}=\left(-n_{2}, n_{1}\right) .
$$

Since $n$ and $n^{\perp}$ are linearly independent it follows that $\delta$ and $a$ are continuous on $M$.

(b) In three dimensions

$$
\left[S_{h}\right] n=[\delta] n-[a] \times n
$$

It follows that $\delta$ is continuous and $\left[a_{\tan }\right]=0$ (the jump in the tangential components of $a$ vanishes).

2. Tensors in $Z_{h}^{\text {sym }}$ are divergence free which restricts the jumps in the derivatives of $\delta$ and $a$.

(a) In two dimensions $\operatorname{div}\left(S_{h}\right)=\nabla \delta-(\nabla a)^{\perp}$ on each element. Cross differentiating shows $\Delta \delta=\Delta a=0$ on each finite element of $M$,

Also, $[\nabla \delta]=\left[(\nabla a)^{\perp}\right]$ along an edge between two finite element of $M$. Then

$$
[\nabla \delta] . n=\left[(\nabla a)^{\perp}\right] . n=\partial[a] / \partial e=0,
$$

where $\partial[a] / \partial e$ denotes the derivative of $[a]$ along the edge. It follows that $[\nabla \delta] . n=0$ so $\delta \in C^{1}(M)$ and similarly $a \in C^{1}(M)$ so $\delta$ and $a$ are harmonic on $M$, and hence smooth. For the usual finite element spaces this requires $a$ and $\delta$ each to be harmonic polynomials on $M$. 
(b) In three dimensions, $\operatorname{div}\left(S_{h}\right)=\nabla \delta+\operatorname{curl}(a)$ on each finite element. Cross differentiation shows $\Delta \delta=$ $\operatorname{curl}(\operatorname{curl}(a))=0$ on each finite element of $M$. Also $[\nabla \delta]=[\operatorname{curl}(a)]$ on a face $k$ between two finite elements of $M$. Stokes' theorem shows

$$
\int_{k}-[\nabla \delta] \cdot n \mathrm{~d} a=\int_{k}[\operatorname{curl}(a)] \cdot n \mathrm{~d} a=\int_{\partial k}[a] \cdot \mathrm{d} s=0,
$$

since $a$ is continuous at the edges (they are tangent to the faces). If $\delta$ is piecewise linear then $[\nabla \delta] . n=0$ so $\delta \in C^{1}(M)$ is smooth.

3. Functions in $Z_{h}^{\text {sym }}$ are orthogonal to $\mathbb{G}_{h}^{\text {skw }}$, thus if this space is sufficiently rich to annihilate $W(a)$ when $a$ is as above, conclude $a=0$. Then $\nabla \delta=(\nabla a)^{\perp}=0(2 \mathrm{~d})$ or $\nabla \delta=-\operatorname{curl}(a)=0(3 \mathrm{~d})$; and in either case $\delta$ is constant.

In three dimensions the last step requires $\mathbb{G}_{h}^{\text {skw }}$ to annihilate a much larger collection of (vector valued) functions, $a$, and fails for many elements developed for the elasticity problem with weak symmetry.

\subsection{Construction of finite elements}

In this section the macroelement methodology is used to develop finite element triples satisfying Assumption 3.1. Two elements will be developed for the two dimensional problem using well-known elements for the elasticity problem with weak symmetry; counter examples show the analogous construction fails in three dimensions. Subsequently a new family of elements is developed which provides both two and three dimensional elements satisfying Assumption 3.1.

The following notation is adopted for the classical finite element spaces.

Notation 4.4. Let $\left\{\mathcal{T}_{h}\right\}_{h>0}$ be a family of triangulations of a domain $\Omega \subset \mathbb{R}^{d}$.

1. If $M \subset \mathcal{T}_{h}$,

$$
\begin{aligned}
& \mathcal{P}_{k}^{\text {cont }}(M)=\left\{p_{h} \in C(M)\left|p_{h}\right|_{K} \in \mathcal{P}_{k}(K), K \in M\right\} \quad \text { and } \\
& \mathcal{P}_{k}^{\text {disc }}(M)=\left\{p_{h} \in L^{2}(M)\left|p_{h}\right|_{K} \in \mathcal{P}_{k}(K), K \in M\right\}
\end{aligned}
$$

denote the spaces of continuous and discontinuous piecewise polynomials of degree $k$ on $M$ respectively. Vectors with components in these spaces will be denoted $\mathcal{P}_{k}^{\text {cont }}(M)^{d}$ and $\mathcal{P}_{k}^{\text {disc }}(M)^{d}$, and $d \times d$ tensors with polynomial components are defined similarly, and

$$
\mathcal{P}_{k}^{\text {cont }}(M)_{\mathrm{skw}}^{d \times d} \quad \text { and } \quad \mathcal{P}_{k}^{\text {disc }}(M)_{\mathrm{sym}}^{d \times d}
$$

denote the skew and symmetric subspaces.

2. If $M \subset \mathcal{T}_{h}$ then $R T_{k}(M) \subset H(\operatorname{div} ; M)$ and $B D M_{k}(M) \subset H(\operatorname{div} ; M)$ denote the subspaces of tensor valued functions with rows in the classical Raviart-Thomas space of order $k$ [21] and Brezzi-Douglas-Marini space of degree $k[7]$.

3. The bubble function on $\mathcal{T}_{h}$ is denoted by $b$; this function is piecewise cubic when $d=2$ and quartic when $d=3$.

\subsubsection{Augmented PEERS element}

In this section we augment the two dimensional PEERS element of Arnold, Brezzi, and Douglas [1] with a suitable class of symmetric matrices to obtain a triple satisfying Assumption 3.1. A counterexample shows that this construction fails in three dimensions.

Lemma 4.5. Let $\mathcal{T}_{h}$ be a triangulation of a bounded Lipschitz domain $\Omega \subset \mathbb{R}^{2}$ and let

$$
\begin{aligned}
& \mathbb{G}_{h}=\mathbb{G} \cap\left(\mathcal{P}_{1}^{\text {cont }}\left(\mathcal{T}_{h}\right)_{\mathrm{skw}}^{2 \times 2} \oplus \mathcal{P}_{1}^{\text {disc }}\left(\mathcal{T}_{h}\right)_{\mathrm{sym}}^{2 \times 2}\right), \\
& \mathbb{U}_{h}=\mathcal{P}_{0}^{\text {disc }}\left(\mathcal{T}_{h}\right)^{2}, \\
& \mathbb{S}_{h}=\mathbb{S} \cap\left(R T_{0}\left(\mathcal{T}_{h}\right) \oplus \mathcal{P}_{0}^{\text {disc }}\left(\mathcal{T}_{h}\right)^{2} \otimes(\nabla b)^{\perp}\right) .
\end{aligned}
$$

Then the triple $\mathbb{G}_{h} \times \mathbb{U}_{h} \times \mathbb{S}_{h}$ satisfies Assumption 3.1 with constant depending only upon the aspect ratio of $\mathcal{T}_{h}$. 
Remark 4.6. The PEERS finite element space is $\left(\mathbb{G} \cap \mathcal{P}_{1}^{\text {cont }}\left(\mathcal{T}_{h}\right)_{\mathrm{skw}}^{2 \times 2}\right) \times \mathbb{U}_{h} \times \mathbb{S}_{h}$.

Proof. Let a typical macroelement be the set of triangles containing a specified vertex $x_{0}$ interior to $\Omega$. On each triangle the functions in $\mathbb{S}_{h}$ take the form

$$
S_{h}(x)=A+\alpha \otimes x+\psi \otimes(\nabla b(x))^{\perp}, \quad A \in \mathbb{R}^{2 \times 2}, \alpha, \psi \in \mathbb{R}^{2},
$$

and $\operatorname{div}\left(S_{h}\right)=\alpha$. Since the average of $\nabla b$ vanishes on $K$, the divergence free tensors orthogonal to the piecewise constant trace-free matrices take the form

$$
S_{h}=\delta I+W(a)+\psi \otimes(\nabla b)^{\perp}, \quad \delta, a \in \mathbb{R}, \psi \in \mathbb{R}^{2} .
$$

An elementary calculation shows that if $S_{h}$ is also orthogonal to $\mathbb{G}_{h}^{\text {sym }}$ then $\psi=0$. Arguing as in steps (1) and (2) above shows $\delta$ and $a$ are constant functions on $M$. The space $\mathbb{G}_{h}^{\text {skw }}$ contains $W(\phi)$ where $\phi$ is the piecewise linear "hat" function on $M$. Then

$$
0=\int_{M} W(a): W(\phi)=2 a \int_{M} \phi=(2|M| / 3) a,
$$

shows $a=0$.

The following example shows that in three dimensions the subspace $Z_{h}^{\mathrm{sym}}$ constructed from the PEERS element contains non-vanishing skew-symmetric tensors so the inf-sup condition can not hold. This is closely related to the property that the continuous $\mathcal{P}_{1}^{d} \times \mathcal{P}_{1}$ space is not div-stable.

Example 4.7. Given a triangulation $\mathcal{T}_{h}$ of a domain $\Omega \subset \mathbb{R}^{3}$ let $p_{h} \in \mathcal{P}_{1}\left(\mathcal{T}_{h}\right) \cap H_{0}^{1}(\Omega)$ be piecewise linear on $\mathcal{T}_{h}$ and let $S_{h}=W\left(\nabla p_{h}\right)$. Then $S_{h} \in R T_{1}\left(\mathcal{T}_{h}\right)$ and is skew.

The PEERS space has $\mathbb{G}^{\text {skw }}=\mathcal{P}_{1}^{\text {cont }}\left(\mathcal{T}_{h}\right)_{\text {skw }}^{d \times d}$, so $S_{h} \in Z^{\text {sym }}$ if

$$
0=-\int_{\Omega} \nabla p_{h} \cdot v_{h}=\int_{\Omega} p_{h} \operatorname{div}\left(v_{h}\right) \quad v_{h} \in \mathcal{P}_{1}^{\text {cont }}\left(\mathcal{T}_{h}\right)^{d} .
$$

Notice that $\operatorname{div}\left(v_{h}\right) \subset \mathcal{P}_{0}\left(\mathcal{T}_{h}\right)$ and this later space has dimension equal to the number of tetrahedra in $\mathcal{T}_{h}$ which we denote by $t$. The inf-sup condition will then fail if we show that the dimension of $\mathcal{P}_{1}\left(\mathcal{T}_{h}\right) \cap H_{0}^{1}(\Omega)$, namely the number of internal vertices of $\mathcal{T}_{h}$, is larger than $t$.

Recall that Euler's formula states $t-f+e-v=O(1)$, where $t, f, e$, and $v$, denote the number of tetrahedra, triangular faces, edges, and vertices of $\mathcal{T}_{h}$. Since each tetrahedron has four faces and each (interior) face is the intersection of two tetrahedra it follows that $4 t \simeq 2 f$. Similarly, $2 e=\bar{d} v$ where $\bar{d}$ is the average degree of the vertices in $\mathcal{T}_{h}$. It follows that

$$
t \simeq v-e=(1-2 / \bar{d}) v .
$$

This formula is asymptotically correct for large $v$ since the boundary contains $O\left(v^{2 / 3}\right)$ vertices. Thus for large meshes the skew subspace of $Z_{h}^{\text {sym }}$ has dimension at least $O((2 / \bar{d}) v)$.

\subsubsection{Augmented AFW element}

In this section we augment the two dimensional Arnold-Falk-Winther element [3] with a suitable class of symmetric matrices to obtain a triple satisfying Assumption 3.1. A counterexample shows that this construction fails in three dimensions.

Lemma 4.8. Let $\mathcal{T}_{h}$ be a triangulation of a bounded Lipschitz domain $\Omega \subset \mathbb{R}^{2}$ and let

$$
\begin{aligned}
& \mathbb{G}_{h}=\mathbb{G} \cap\left(\mathcal{P}_{0}^{\text {disc }}\left(\mathcal{T}_{h}\right)_{\mathrm{skw}}^{2 \times 2} \oplus \mathcal{P}_{1}^{\text {disc }}\left(\mathcal{T}_{h}\right)_{\mathrm{sym}}^{2 \times 2}\right), \\
& \mathbb{U}_{h}=\mathcal{P}_{0}^{\text {disc }}\left(\mathcal{T}_{h}\right)^{2}, \\
& \mathbb{S}_{h}=\mathbb{S} \cap B D M_{1}\left(\mathcal{T}_{h}\right) .
\end{aligned}
$$

Then the triple $\mathbb{G}_{h} \times \mathbb{U}_{h} \times \mathbb{S}_{h}$ satisfies Assumption 3.1 with constant depending only upon the aspect ratio of $\mathcal{T}_{h}$. 
Remark 4.9. The AFW finite element space is $\left(\mathbb{G} \cap \mathcal{P}_{0}^{\text {disc }}\left(\mathcal{T}_{h}\right)_{\text {skw }}^{2 \times 2}\right) \times \mathbb{U}_{h} \times \mathbb{S}_{h}$.

Proof. Let the macroelements consist of a non-boundary triangle and the three triangles adjacent to it. On each triangle the functions in $\mathbb{S}_{h}$ are piecewise linear, so it is immediate that the functions orthogonal to $\mathcal{P}_{1}^{\text {disc }}\left(\mathcal{T}_{h}\right)_{\text {sym }}^{2 \times 2}$ take the form

$$
S_{h}=\delta I+W(a) \quad \delta, a \in \mathcal{P}_{1}^{\operatorname{disc}}(M) .
$$

Arguing as in steps (1) and (2) above, it follows that $a$ and $\delta$ are smooth, so they must be linear polynomials on $M$. If, in addition, $S_{h}$ is orthogonal to $\mathbb{G}_{h}^{\text {skw }}$, it follows that

$$
0=\int_{K} a_{0}+a_{1} x+a_{2} y=a_{0}+\left(a_{1}, a_{2}\right) \cdot \bar{x}_{K}, \quad K \subset M,
$$

where we have written $a(x, y)=a_{0}+a_{1} x+a_{2} y$, and $\bar{x}_{K}$ denotes the centroid of $K$. If $a(x, y)$ is non-zero it follows that the four centroids of the triangles $K \subset M$ lie on the line $0=a_{0}+a_{1} x+a_{2} y$ which is impossible. A proof of this intuitively obvious geometric property is given in the Appendix.

Example 4.10. Given a triangulation $\mathcal{T}_{h}$ of a domain $\Omega \subset \mathbb{R}^{3}$, let $p_{h} \in \mathcal{P}_{2}^{\text {cont }}\left(\mathcal{T}_{h}\right)$ be piecewise quadratic and let $S_{h}=W\left(\nabla p_{h}\right)$. Then $S_{h} \in B D M_{1}\left(\mathcal{T}_{h}\right)$ and is skew.

The augmented AFW space has $\mathbb{G}^{\text {skw }}=\mathcal{P}_{0}^{\text {disc }}\left(\mathcal{T}_{h}\right)_{\text {skw }}^{d \times d}$, so $S_{h} \in Z^{\text {sym }}$ if

$$
0=-\int_{\Omega} \nabla p_{h} . u_{h}=\sum_{k} \int_{k} p_{h}\left[u_{h} . n\right] \quad u_{h} \in \mathcal{P}_{0}^{\mathrm{disc}}\left(\mathcal{T}_{h}\right)^{d},
$$

where the sum is over the (triangular) faces in $\mathcal{T}_{h}$ and $n$ denotes their normal. In this formula $\left[u_{h} . n\right] \equiv u_{h} . n$ for faces on the boundary.

If $k$ is a triangle and the mid points of the edges are denoted by $\left\{\bar{x}_{1}^{k}, \bar{x}_{2}^{k}, \bar{x}_{3}^{k}\right\}$, then the quadrature rule

$$
\int_{k} f=(|k| / 3)\left(f\left(\bar{x}_{1}^{k}\right)+f\left(\bar{x}_{2}^{k}\right)+f\left(\bar{x}_{3}^{k}\right)\right)
$$

is exact on $\mathcal{P}_{2}(k)$. It follows that $S_{h} \in Z_{h}^{\text {sym }}$ if $p_{h}\left(\bar{x}_{i}\right)=0$ at the mid points of the edges in $\mathcal{T}_{h}$. Upon recalling that the degrees of freedom for the piecewise quadratic finite element space are the function values at the vertices and at the mid points of the edges, it follows that the skew subspace of $Z_{h}^{\text {sym }}$ has dimension at least as large as the number of vertices in $\mathcal{T}_{h}$.

\subsection{A new family of elements in 2 and 3 dimensions}

In this section we construct a family of composite elements that satisfy Assumption 3.1, using the div-stable elements of Scott and Vogelius [22,28]. We make use of the following result which was proved in [5], Theorem 9.1 in two dimensions and in [6], Proposition 4, when $d=3$.

Theorem 4.11. Let $\left(\mathbb{U}_{h}, \mathbb{S}_{h}\right) \subset L^{2}(\Omega)^{d} \times H(\Omega ; \operatorname{div})$ be a div-stable pair of spaces,

$$
\inf _{u_{h} \in U_{h}} \sup _{S_{h} \in \mathbb{S}_{h}} \frac{\left(u_{h}, \operatorname{div}\left(S_{h}\right)\right)}{\left\|S_{h}\right\|_{H(\Omega ; \operatorname{div})}\left\|u_{h}\right\|_{L^{2}(\Omega)}} \geq c \quad \text { and } \quad \operatorname{div}\left(\mathbb{S}_{h}\right) \subset \mathbb{U}_{h} .
$$

If $V_{h}^{d} \times P_{h} \subset H_{0}^{1}(\Omega)^{d} \times L^{2}(\Omega) / \mathbb{R}$ is a div-stable velocity-pressure space for the Stokes problem and $\operatorname{Curl}\left(V_{h}\right) \subset \mathbb{S}_{h}$, then $W\left(P_{h}\right) \times \mathbb{U}_{h} \times \mathbb{S}_{h}$ is a stable triple for the elasticity problem with weak symmetry.

Augmenting the spaces constructed in this theorem using Raviart-Thomas and Scott-Vogelius elements gives a family of spaces satisfying Assumption 3.1. 


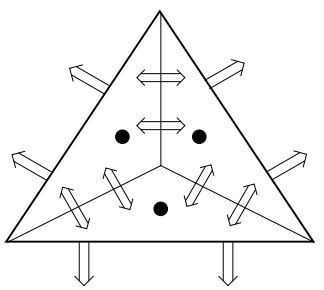

(a) $\mathbb{S}_{h}$ on $K^{r}$

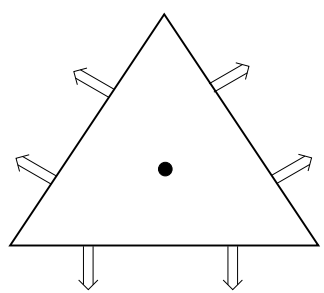

(b) Condensed $\mathbb{S}_{h}$

FiguRe 1. Degrees of freedom for the lowest order $(k=1)$ element in two space dimensions, which consists of two rows, each of which are first-order Raviart-Thomas vectors. The double arrows represent continuity of the normal components of each row of $S_{h}$, and the dot represents the four internal degrees of freedom of $S_{h}$ (two per row). The diagram on the left represents $\mathbb{S}_{h}$ on a barycentric refined mesh, and the diagram on the right represents the condensed element.

Lemma 4.12. Let $k \geq 1$ be an integer and let $\mathcal{T}_{h}$ be a triangulation of a bounded Lipschitz domain $\Omega \subset \mathbb{R}^{d}$, $d=2$ or $d=3$, and let $\mathcal{T}_{h}^{r}$ denote the barycentric refinement of $\mathcal{T}_{h}$ and

$$
\begin{aligned}
& \mathbb{G}_{h}=\mathbb{G} \cap \mathcal{P}_{k}^{\text {disc }}\left(\mathcal{T}_{h}^{r}\right)^{d \times d}, \\
& \mathbb{U}_{h}=\mathcal{P}_{k}^{\text {disc }}\left(\mathcal{T}_{h}^{r}\right)^{d}, \\
& \mathbb{S}_{h}=\mathbb{S} \cap R T_{k}\left(\mathcal{T}_{h}^{r}\right) .
\end{aligned}
$$

If $k \geq 1$ when $d=2$ or $k \geq 2$ when $d=3$ the triple $\mathbb{G}_{h} \times \mathbb{U}_{h} \times \mathbb{S}_{h}$ satisfies Assumption 3.1 with constant depending only upon the aspect ratio of $\mathcal{T}_{h}$.

Proof. Under the assumptions stated on $k$, the Scott-Vogelius space $\left(V_{h}^{d}, P_{h}\right) \equiv \mathcal{P}_{k+1}^{\text {cont }}\left(\mathcal{T}_{h}^{r}\right)^{d} \times \mathcal{P}_{k}^{\text {disc }}\left(\mathcal{T}_{h}^{r}\right)$ is a divstable element for the Stokes problem [28]; moreover, $\operatorname{Curl}\left(V_{h}\right) \subset R T_{k}\left(\mathcal{T}_{h}^{r}\right)$ since (i) functions in $\operatorname{Curl}\left(V_{h}\right)$ belong to $H(\Omega ; \operatorname{div})$, and (ii) the Raviart-Thomas spaces contain all piecewise polynomials of degree $k$ in $H(\Omega ; \operatorname{div})$. It follows that $W\left(P_{h}\right) \times \mathbb{U}_{h} \times \mathbb{S}_{h}$ is a stable triple for the elasticity problem with weak symmetry.

Upon recalling that the divergence free functions in $R T_{k}$ are piecewise polynomials of degree $k$, it follows that functions in $Z^{\text {sym }}$ are symmetric (pointwise), and the inf-sup condition in Assumption 3.1 follows upon setting $G_{h}^{\text {sym }}$ to be the trace-free part of $S_{h}$ and using Lemma 2.3 to bound the trace.

Condensing out internal degrees of freedom from composite elements significantly reduces the number of unknowns. The following example illustrates this for the lowest order two dimensional element.

Example 4.13. If $K \in \mathcal{T}_{h}$ is a triangle and $K^{r}$ is its barycentric refinement, the lowest order two dimensional element $(k=1)$ would have (see Fig. 1)

$$
\operatorname{dim}\left(\mathbb{G}_{h}\left(K^{r}\right)\right)=27, \quad \operatorname{dim}\left(\mathbb{U}_{h}\left(K^{r}\right)\right)=18, \quad \operatorname{dim}\left(\mathbb{S}_{h}\left(K^{r}\right)\right)=36 .
$$

Consider then the two subspaces of weakly symmetric tensors on $K^{r}$

$$
\begin{aligned}
\mathbb{S}_{h}^{\text {sym }}\left(K^{r}\right) & =\left\{S_{h} \in \mathbb{S}_{h}\left(K^{r}\right) \mid \int_{K} S_{h}: G_{h}=0, G_{h} \in \mathbb{G}_{h}\left(K^{r}\right)^{\text {skw }}\right\}, \\
\overline{\mathbb{S}}_{h}(K) & =\left\{S_{h} \in \mathbb{S}_{h}^{\mathrm{sym}}\left(K^{r}\right) \mid \operatorname{div}\left(S_{h}\right) \in \mathcal{P}_{1}(K)\right\} .
\end{aligned}
$$

Then $\overline{\mathbb{S}}_{h}(K)$ has dimension 15 and a set of degrees of freedom almost identical to to $R T_{1}(K)$; namely, the trace of $S n$ on each edge of $K$ and the average of the symmetric part of $S_{h}$ over $K$ (the average of the skew part being zero), see Figure 1. This gives rise to a decomposition

$$
\mathbb{S}_{h}^{\text {sym }}\left(K^{r}\right)=\overline{\mathbb{S}}_{h}(K)+\mathbb{S}_{h}^{0}\left(K^{r}\right), \quad \mathbb{S}_{h}^{0}\left(K^{r}\right)=\left\{S_{h} \in \mathbb{S}_{h}\left(K^{r}\right) \mid S_{h} n=0 \text { on } \partial K\right\} .
$$


The degrees of freedom for $\mathbb{S}_{h}^{0}\left(K^{r}\right)$ would be the ones illustrated in Figure 1 which are interior to $K$.

Let

$$
\overline{\mathbb{U}}_{h}=\mathcal{P}_{1}^{\text {disc }}\left(\mathcal{T}_{h}\right)^{2} \quad \text { and } \quad \overline{\mathbb{S}}_{h}=\mathbb{S} \cap\left\{S_{h} \in H(\Omega ; \operatorname{div})\left|S_{h}\right|_{K} \in \overline{\mathbb{S}}_{h}(K), K \in \mathcal{T}_{h}\right\}
$$

Then solutions of the Navier-Stokes problem would seek $\left(\bar{u}_{h}, \bar{S}_{h}\right) \in \overline{\mathbb{U}}_{h} \times \overline{\mathbb{S}}_{h}$ such that

$$
\begin{gathered}
\int_{\Omega} G_{h} u_{h} \cdot \bar{v}_{h}-\operatorname{div}\left(\bar{S}_{h}+S_{h}^{0}\right) \cdot \bar{v}_{h}=\int_{\Omega} f \cdot \bar{v}_{h}, \quad \bar{v}_{h} \in \overline{\mathbb{U}}_{h} \\
\int_{\Omega} G_{h}: \bar{T}_{h}+\bar{u}_{h} \cdot \operatorname{div}\left(\bar{T}_{h}\right)=0, \quad \bar{T}_{h} \in \overline{\mathbb{S}}_{h},
\end{gathered}
$$

where on each element

$$
\left(G_{h}, u_{h}, S_{h}^{0}\right) \in \mathcal{P}_{1}^{\text {disc }}\left(K^{r}\right)^{2 \times 2} \times \mathcal{P}_{1}^{\text {disc }}\left(K^{r}\right)^{2} \times \mathbb{S}_{h}^{0}\left(K^{r}\right)
$$

are determined from $\left(\bar{u}_{h}, \bar{S}_{h}\right)$ as the solution of the local problem:

$$
\begin{gathered}
\int_{K} \mathcal{A}\left(G_{h}\right): H_{h}-(1 / 2)\left(u_{h} \otimes u_{h}\right): H_{h}-\left(\bar{S}+S_{h}^{0}\right): H_{h}=0, \quad H_{h} \in \mathcal{P}_{1}^{\mathrm{disc}}\left(K^{r}\right)^{2 \times 2} \\
\int_{K} G_{h} u_{h} \cdot v_{h}-\operatorname{div}\left(\bar{S}_{h}+S_{h}^{0}\right) \cdot v_{h}=\int_{\Omega} f \cdot v_{h}, \quad v_{h} \in \mathcal{P}_{1}^{\mathrm{disc}}\left(K^{r}\right)^{2} \\
\int_{K} G_{h}: T_{h}^{0}+u_{h} \cdot \operatorname{div}\left(T_{h}^{0}\right)=0, \quad T_{h}^{0} \in \mathbb{S}_{h}^{0}\left(K^{r}\right), \\
\int_{K} u_{h} \cdot \bar{v}_{h}=\int_{K} \bar{u}_{h} \cdot \bar{v}_{h}, \quad \bar{v}_{h} \in \mathcal{P}_{1}^{\mathrm{disc}}\left(K^{r}\right)^{2} .
\end{gathered}
$$

The last equation can be eliminated if a basis for the orthogonal decomposition $\mathcal{P}_{1}^{\text {disc }}\left(K^{r}\right)^{2}=\mathcal{P}_{1}^{\text {disc }}(K)^{2} \oplus$ $\left(\mathcal{P}_{1}^{\text {disc }}(K)^{2}\right)^{\perp}$ is available.

\section{Numerical EXAmples}

The following non-homogeneous solution of the two dimensional Navier-Stokes equations is the stationary analog of the solution from [23].

$$
\begin{aligned}
& u=((-m / k) \sin (k x) \cos (m y), \cos (k x) \sin (m y))^{T} \\
& p=(-1 / 2)\left(|u|^{2}+\left(1-(m / k)^{2}\right) \sin ^{2}(k x) \sin ^{2}(m y)\right)
\end{aligned}
$$

with right hand side $f=\nu\left(k^{2}+m^{2}\right) u$. The computational domain was chosen to be $\Omega=(-1,1)^{2}$, the traction boundary condition was specified on the right edge $(x=1)$, and Dirichlet data was specified on the remainder of the boundary. Triangulations were formed by sub-dividing the square uniformly into squares of edge length $h=2 / N$ and dividing each of these into two triangles. The parameters were selected to be $k=\pi, m=\pi / 2$, and $\nu=1 / 20$.

Figures 1 and 2 tabulate the errors of the approximate solutions computed using the dual-mixed formulation with the augmented AFW and new element respectively. A first order rate of convergence for the augmented AFW element is observed and a second order rate for the new element is achieved for the finer meshes.

\section{Appendix A. Collinearity of triangle Centroids}

The following lemma was used in the proof of Lemma 4.8.

Lemma A.1. Let $\left\{K_{i}\right\}_{i=0}^{3}$ be triangles in the plane with disjoint interiors and let each of $K_{1}$, $K_{2}$, and $K_{3}$ have an edge in common with $K_{0}$. Then the centroids of the four triangles are not collinear. 
TABLE 1. Errors for the dual-mixed formulation of the Navier-Stokes problem using the augmented AFW element.

\begin{tabular}{|l|ccccc|}
\hline$h$ & $G^{\text {sym }}$ & $G^{\text {skw }}$ & $u$ & $S$ & $\operatorname{div}(S)$ \\
\hline $1 / 4$ & $6.883930 \mathrm{e}-01$ & $6.544852 \mathrm{e}-01$ & $2.312414 \mathrm{e}-01$ & $1.505661 \mathrm{e}-01$ & $2.405736 \mathrm{e}-01$ \\
$1 / 8$ & $3.314210 \mathrm{e}-01$ & $3.269643 \mathrm{e}-01$ & $1.157281 \mathrm{e}-01$ & $6.841425 \mathrm{e}-02$ & $1.205427 \mathrm{e}-01$ \\
$1 / 16$ & $1.637091 \mathrm{e}-01$ & $1.631461 \mathrm{e}-01$ & $5.785624 \mathrm{e}-02$ & $3.320099 \mathrm{e}-02$ & $6.013830 \mathrm{e}-02$ \\
$1 / 32$ & $8.157049 \mathrm{e}-02$ & $8.150047 \mathrm{e}-02$ & $2.892592 \mathrm{e}-02$ & $1.646940 \mathrm{e}-02$ & $3.004137 \mathrm{e}-02$ \\
$1 / 64$ & $4.074540 \mathrm{e}-02$ & $4.073677 \mathrm{e}-02$ & $1.446263 \mathrm{e}-02$ & $8.218179 \mathrm{e}-03$ & $1.501600 \mathrm{e}-02$ \\
$1 / 128$ & $2.036695 \mathrm{e}-02$ & $2.036590 \mathrm{e}-02$ & $7.231270 \mathrm{e}-03$ & $4.107087 \mathrm{e}-03$ & $7.507253 \mathrm{e}-03$ \\
\hline Norm & 2.776802 & 2.776802 & 1.118034 & 0.905688 & 0.927988 \\
\hline Rate & 1.0135 & 1.0013 & 0.9999 & 1.0333 & 1.0008 \\
\hline
\end{tabular}

TABLE 2. Errors for the dual-mixed formulation of the Navier-Stokes problem using the new element (rate for $h \in\{1 / 16,1 / 32,1 / 64,1 / 128\}$ ).

\begin{tabular}{|l|ccccc|}
\hline$h$ & $G^{\text {sym }}$ & $G^{\text {skw }}$ & $u$ & $S$ & $\operatorname{div}(S)$ \\
\hline $1 / 4$ & $2.451267 \mathrm{e}-01$ & $4.576332 \mathrm{e}-01$ & $2.399623 \mathrm{e}-02$ & $3.780692 \mathrm{e}-02$ & $9.647025 \mathrm{e}-02$ \\
$1 / 8$ & $8.182080 \mathrm{e}-02$ & $2.012711 \mathrm{e}-01$ & $5.839852 \mathrm{e}-03$ & $1.210886 \mathrm{e}-02$ & $4.150840 \mathrm{e}-02$ \\
$1 / 16$ & $2.414153 \mathrm{e}-02$ & $7.073387 \mathrm{e}-02$ & $1.284591 \mathrm{e}-03$ & $3.530203 \mathrm{e}-03$ & $1.453778 \mathrm{e}-02$ \\
$1 / 32$ & $6.452742 \mathrm{e}-03$ & $2.032373 \mathrm{e}-02$ & $2.863796 \mathrm{e}-04$ & $9.407521 \mathrm{e}-04$ & $4.194951 \mathrm{e}-03$ \\
$1 / 64$ & $1.650640 \mathrm{e}-03$ & $5.324535 \mathrm{e}-03$ & $6.817378 \mathrm{e}-05$ & $2.404370 \mathrm{e}-04$ & $1.103307 \mathrm{e}-03$ \\
$1 / 128$ & $4.159781 \mathrm{e}-04$ & $1.352113 \mathrm{e}-03$ & $1.679452 \mathrm{e}-05$ & $6.057340 \mathrm{e}-05$ & $2.807632 \mathrm{e}-04$ \\
\hline Norm & 2.776802 & 2.776802 & 1.118034 & 0.905688 & 0.927988 \\
\hline Rate & 1.9543 & 1.9060 & 2.0842 & 1.9563 & 1.9010 \\
\hline
\end{tabular}

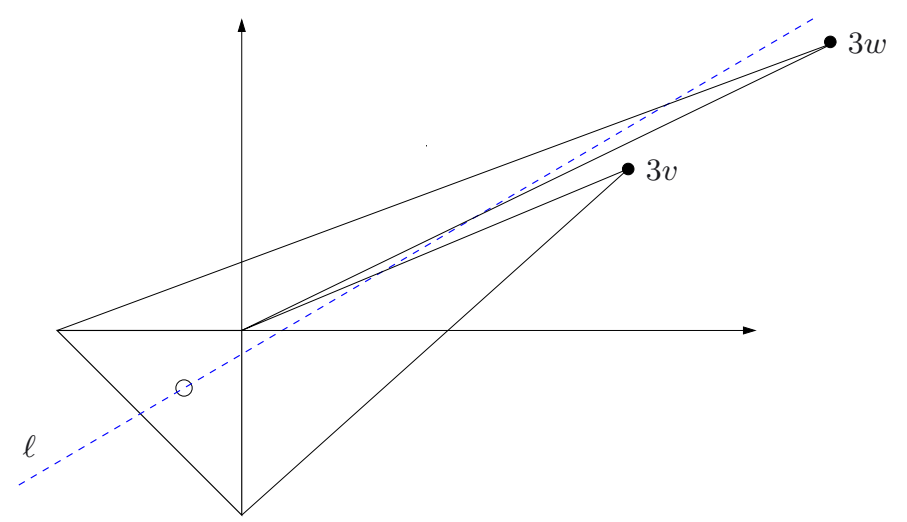

Figure 2. Centroids of the three triangles can not lie on the dashed line.

Proof. To obtain a contradiction, let $\ell$ be a line containing all four centroids, then two of the three centroids of $K_{1}, K_{2}$ and $K_{3}$ lie on one side of the centroid of $K_{0}$. Since averages map to averages under affine maps, it suffices to consider the situation where $K_{0}$ is the triangle with coordinates $\left(0,-3 e_{1},-3 e_{2}\right)$ and and the centroids of the triangles sharing the top and right edges of $K_{0}$ have their centroids on the same side of $\ell$. Assume without loss of generality that $\ell$ exits $K_{0}$ on the right, so that it has slope less than 1 (otherwise reflect about the line $y=x)$.

Let the top triangle have vertex $3 w$ and the triangle on the right have vertex $3 v$, so that the centroids are

$$
c_{\text {top }}=(1 / 3)\left(0+3 w-3 e_{1}\right)=w-e_{1}, \quad \text { and } \quad c_{\text {right }}=(1 / 3)\left(0+3 v-3 e_{2}\right)=v-e_{2} .
$$


Since the top triangle (a) has all its vertices above the $x$-axis and (b) intersects $\ell$, it follows that $w$ is in the positive quadrant, as shown in Figure 2.

If the centroids $c_{\text {top }}, c_{\text {right }}$ and $c=(-1,-1)$ of $K_{0}$ are collinear, there exists $\lambda>0$ such that the equation

$$
\lambda\left(c_{\text {top }}-c\right)=c_{\text {right }}-c, \text { i.e. } \quad \lambda\left(w-e_{1}-c\right)=v-e_{2}-c,
$$

has a solution with $w$ in the positive quadrant, $\lambda>0$ and $v_{1}>0$. Writing out the two components of this shows

$$
\left.\begin{array}{c}
\lambda w_{1}=v_{1}+1 \\
\lambda\left(w_{2}+1\right)=v_{2}
\end{array}\right\} \quad \Rightarrow \quad w_{1} v_{2}=\left(v_{1}+1\right)\left(w_{2}+1\right) .
$$

Note that $v_{1}>0$ and the second equation shows $v_{2}>0$, so $v$ is also in the positive quadrant.

Next, geometric consistency (triangle interiors do not intersect) requires that the angle $w$ forms with the $x$-axis to be greater than the angle $v$ forms;

$$
w_{2} / w_{1} \geq v_{2} / v_{1}, \quad \text { or } \quad v_{1} w_{2} \geq w_{1} v_{2} .
$$

Expanding the equation $w_{1} v_{2}=\left(v_{1}+1\right)\left(w_{2}+1\right)$ shows

$$
w_{1} v_{2}-v_{1} w_{2}=1+v_{1}+w_{2},
$$

and the inequality $0 \geq w_{1} v_{2}-v_{1} w_{2}$ shows no solution exits.

\section{REFERENCES}

[1] D.N. Arnold, F. Brezzi and J. Douglas, Jr., PEERS: a new mixed finite element for plane elasticity. Japan J. Appl. Math. 1 (1984) 347-367.

[2] D.N. Arnold, J. Douglas, Jr. and C.P. Gupta, A family of higher order mixed finite element methods for plane elasticity. Numer. Math. 45 (1984) 1-22.

[3] D.N. Arnold, R.S. Falk and R. Winther, Mixed finite element methods for linear elasticity with weakly imposed symmetry. Math. Comput. 76 (2007) 1699-1723 (electronic).

[4] J. Barlow, Optimal stress location in finite element method. Internat. J. Numer. Methods Engrg. 10 (1976) $243-251$.

[5] D. Boffi, F. Brezzi, L.F. Demkowicz, R.G. Durán, R.S. Falk and M. Fortin, Mixed finite elements, compatibility conditions, and applications, Springer-Verlag, Berlin. Lect. Notes Math. 1939 (2008). Lectures given at the C.I.M.E. Summer School held in Cetraro, June 26-July 1, 2006, edited by Boffi and Lucia Gastaldi.

[6] D. Boffi, F. Brezzi and M. Fortin, Reduced symmetry elements in linear elasticity. Commun. Pure Appl. Anal. 8 (2009) 95-121.

[7] F. Brezzi, J. Douglas, Jr. and L.D. Marini, Two families of mixed finite elements for second order elliptic problems. Numer. Math. 47 (1985) 217-235.

[8] F. Brezzi and M. Fortin, Mixed and hybrid finite element methods, Springer Series in Comput. Math. Springer-Verlag, New York 15 (1991).

[9] F. Brezzi, J. Rappaz and P.-A. Raviart, Finite-dimensional approximation of nonlinear problems. I. Branches of nonsingular solutions. Numer. Math. 36 (1980/81) 1-25.

[10] Z. Cai, C. Wang and S. Zhang, Mixed finite element methods for incompressible flow: stationary Navier-Stokes equations. SIAM J. Numer. Anal. 48 (2010) 79-94.

[11] Z. Cai and Y. Wang, Pseudostress-velocity formulation for incompressible Navier-Stokes equations. Int. J. Numer. Methods Fluids 63 (2010) 341-356.

[12] P. Clément, Approximation by finite element functions using local regularization. RAIRO Anal. Numér. 9 (1975) 77-84.

[13] B. Cockburn, J. Gopalakrishnan and J. Guzmán, A new elasticity element made for enforcing weak stress symmetry. Math. Comput. 79 (2010) 1331-1349.

[14] M. Farhloul and H. Manouzi, Analysis of non-singular solutions of a mixed Navier-Stokes formulation. Comput. Methods Appl. Mech. Engrg. 129 (1996) 115-131.

[15] M. Farhloul, S. Nicaise and L. Paquet, A refined mixed finite-element method for the stationary Navier-Stokes equations with mixed boundary conditions. IMA J. Numer. Anal. 28 (2008) 25-45.

[16] M. Farhloul, S. Nicaise and L. Paquet, A priori and a posteriori error estimations for the dual mixed finite element method of the Navier-Stokes problem. Numer. Methods Partial Differ. Equ. 25 (2009) 843-869.

[17] V. Girault and P.A. Raviart, Finite Element Approximation of the Navier Stokes Equations. Springer Verlag, Berlin, Heidelbert, New York. Lect. Notes Math. 749 (1979).

[18] J. Gopalakrishnan and J. Guzmán, A second elasticity element using the matrix bubble, IMA J. Numer. Anal. 32 (2012) $352-372$. 
[19] J.S. Howell and N.J. Walkington, Inf-sup conditions for twofold saddle point problems. Numer. Math. 118 (2011) 663-693.

[20] W. Layton, Introduction to the numerical analysis of incompressible viscous flows, Computational Science \& Engineering, Society for Industrial and Applied Mathematics (SIAM), Philadelphia, PA 6 (2008).

[21] P.-A. Raviart and J.M. Thomas, A mixed finite element method for 2nd order elliptic problems, in Mathematical aspects of finite element methods (Proc. Conf., Consiglio Naz. delle Ricerche (C.N.R.), Rome, 1975), Springer, Berlin. Lect. Notes Math. 606 (1977) 292-315.

[22] L.R. Scott and M. Vogelius, Norm estimates for a maximal right inverse of the divergence operator in spaces of piecewise polynomials. RAIRO Modél. Math. Anal. Numér. 19 (1985) 111-143.

[23] A. Shapiro, The use of an exact solution of the navier-stokes equations in a validation test of a three-dimensional non-hydrostatic numerical model. Mon. Wea. Rev. 121 (1993) 2420-2425.

[24] R.E. Showalter, Monotone operators in Banach space and nonlinear partial differential equations, Mathematical Surveys and Monographs. American Mathematical Society, Providence, RI 49 (1997).

[25] R. Stenberg, Analysis of mixed finite elements methods for the Stokes problem: a unified approach. Math. Comput. 42 (1984) 9-23.

[26] R. Stenberg, A family of mixed finite elements for the elasticity problem. Numer. Math. 53 (1988) 513-538.

[27] R. Temam, Navier-Stokes Equations, North Holland (1977).

[28] S. Zhang, A new family of stable mixed finite elements for the 3D Stokes equations. Math. Comput. 74 (2005) 543-554.

[29] Z. Zhang, Ultraconvergence of the patch recovery technique. Math. Comput. 65 (1996) 1431-1437.

[30] O.C. Zienkiewicz, R. Taylor and J. Too, Reduced integration technique in general analysis of plates and shells. Inter. J. Numer. Methods Engrg. 3 (1971) 275-290.

[31] O.C. Zienkiewicz and J.Z. Zhu, The superconvergent patch recovery and a posteriori error estimates I. The recovery technique. Internat. J. Numer. Methods Engrg. 33 (1992) 1331-1364.

[32] O.C. Zienkiewicz and J.Z. Zhu, The superconvergent patch recovery and a posteriori error estimates. II. Error estimates and adaptivity. Inter. J. Numer. Methods Engrg. 33 (1992) 1365-1382.

[33] O.C. Zienkiewicz and J.Z. Zhu, The superconvergent patch recovery (SPR) and adaptive finite element refinement. Comput. Methods Appl. Mech. Engrg. 101 (1992) 207-224. Reliability in computational mechanics (Kraków 1991). 